\title{
Relations for classical communication capacity and entanglement capability of two-qubit operations
}

\author{
Dominic W. Berry ${ }^{1}$ and Barry C. Sanders ${ }^{1}$ \\ ${ }^{1}$ Department of Physics and Centre for Advanced Computing - Algorithms and Cryptography, \\ Macquarie University, Sydney, New South Wales 2109, Australia
}

(Dated: November 5, 2018)

\begin{abstract}
Bipartite operations underpin both classical communication and entanglement generation. Using a superposition of classical messages, we show that the capacity of a two-qubit operation for error-free entanglement-assisted bidirectional classical communication can not exceed twice the entanglement capability. In addition we show that any bipartite two-qubit operation can increase the communication that may be performed using an ensemble by twice the entanglement capability.
\end{abstract}

PACS numbers: 03.67.Hk, 03.65.Ud, 03.67.Mn

Classical communication can only occur between two systems via an interaction - that is, a bipartite or multipartite operation. Similarly, interactions are essential for generating entanglement between two systems. We establish relations between the capacities for bipartite operations to perform these two tasks.

In the static case there is equality between entanglement and shared classical information. The Schmidt decomposition of a bipartite state $|\Phi\rangle=$ $\sum_{n=1}^{N} \sqrt{\lambda_{n}}\left|\varphi_{n}\right\rangle\left|\chi_{n}\right\rangle$, with $\left|\varphi_{n}\right\rangle$ and $\left|\chi_{n}\right\rangle$ orthonormal bases for the two systems $A$ and $B$, has an entropy of entanglement given by $E=-\sum \lambda_{n} \log _{2} \lambda_{n}$ [1]. This entropy of entanglement is also the mutual information. That is, if system $A$ is measured in the basis $\left|\varphi_{n}\right\rangle$ and system $B$ is measured in the basis $\left|\chi_{n}\right\rangle$, then identical results will be obtained. The two sets of measurement results will then share mutual information $E$. The mutual information, which is classical, equals the entropy of entanglement, which is quantum. Of course, this mutual information cannot be used for communication as the measurement results are obtained in a completely random way, and not on the basis of pre-existing information.

It is known that there is equality for some simple operations. The CNOT operation can increase entanglement by a maximum of one ebit (an ebit is the entanglement contained in one Einstein-Podolsky-Rosen, or EPR, pair). Similarly it is possible to use a CNOT to communicate one bit in each direction simultaneously [2]. Analogously the SWAP gate may produce a maximum of two ebits or perform two bits of communication in both directions simultaneously using superdense coding [3, 何. In these two cases, the classical communication capacity in each direction is identical to the entanglement capability. It is reasonable to hypothesise that this may be true for all two-qubit operations.

To establish relations, it is useful to consider the entanglement that is generated by a superposition of classical messages. We employ this concept to prove an inequality between the entanglement capability and the error-free classical communication capacity. This result is compared with the recent result of Bennett et al. (BHLS)
[5] where the classical communication is not assisted by entanglement; i.e. entanglement is not provided as a "free resource". We then derive initial ensembles such that the communication that can be performed using these ensembles may be increased by the entanglement capability via a two-qubit operation.

We adopt the BHLS terminology, and their manuscript provides an excellent background on the mathematical foundations for studying entanglement capability and classical communication capacity. The maximum increase in entanglement that may be produced by a single implementation of the transform $U$ is denoted $E_{U}$. The case of a single implementation of $U$ is sufficient to establish this bound as it is not possible to generate more entanglement per operation via multiple operations [5]. Here we specifically address the case that entanglement is quantified by the entropy of entanglement. We employ base-2 logarithms so $E_{U}$ is in units of ebits.

In addition, we adopt the BHLS definitions for the asymptotic communication capacities. The bidirectional communication is described by the pair of numbers $\left(R_{\rightarrow}, R_{\leftarrow}\right)$. The pair $\left(R_{\rightarrow}, R_{\leftarrow}\right)$ is said to be "achievable" if, for any $\epsilon>0$, there exists a $t$ such that it is possible to communicate $t R_{\rightarrow}$ bits from Alice to Bob and $t R_{\leftarrow}$ bits from Bob to Alice with fidelity $1-\epsilon$ via $t$ applications of $U$ interspersed with local unitary operations. The explicit mathematical expression for this is given by BHLS. The total capacity is then defined by

$$
C_{+}^{E}=\sup \left\{R_{\rightarrow}+R_{\leftarrow}:\left(R_{\rightarrow}, R_{\leftarrow}\right) \text { is achievable by } U\right\}
$$

Here we use the superscript $E$ to indicate that any arbitrary amount of entanglement is allowed to assist communication. The case where no additional entanglement is allowed to assist communication is denoted by $C_{+}$(we do not consider this case).

Here we consider the case of error-free communication $(\epsilon=0)$ for simplicity. We denote the capacity for errorfree communication by $\bar{C}_{+}^{E}$. It is shown in Ref. [6] that the same results are obtained for imperfect communication in the limit $\epsilon \rightarrow 0$. In order to derive an inequality for $\bar{C}_{+}^{E}$, we consider a quantum superposition of classical messages. This technique is similar to that applied by 
BHLS to derive the inequality $E_{U} \geq C_{+}$. Let us consider a protocol that transmits $n_{a}$ bits from Alice to Bob and $n_{b}$ bits from Bob to Alice, with $t$ uses of $U$. By taking sufficiently large $t$, the total bidirectional communication per operation, $\left(n_{a}+n_{b}\right) / t$, can be made arbitrarily close to $\bar{C}_{+}^{E}$.

We consider an input state $|x\rangle_{A_{1}}|y\rangle_{B_{1}}|\psi\rangle_{A_{2} B_{2}}$, where subsystem $A_{1}$ contains Alice's message $x$ and subsystem $B_{1}$ contains Bob's message $y . A_{2}$ and $B_{2}$ are auxiliary subsystems possessed by Alice and Bob, respectively, and may contain as much entanglement as is necessary in order to perform the entanglement assisted communication. Via $t$ applications of $U$, together with local unitary transformations, we obtain the output state $\left|\eta_{x y}\right\rangle=|y\rangle_{A_{1}}|x\rangle_{B_{1}}\left|c_{x y}\right\rangle_{A_{2} B_{2}}$. That is, the message $y$ is transferred to Alice, $x$ is transferred to Bob, and the auxiliary subsystems $A_{2}$ and $B_{2}$ are left in a state which may, in general, depend on $x$ and $y$.

The change in the entanglement is then

$$
\Delta E_{x y}=S\left[\operatorname{Tr}_{B_{2}}\left(\left|c_{x y}\right\rangle_{A_{2} B_{2}}\right)\right]-S\left[\operatorname{Tr}_{B_{2}}\left(|\psi\rangle_{A_{2} B_{2}}\right)\right]
$$

for $S(\rho)=-\operatorname{Tr}(\rho \log \rho)$, and adopting the convention that $\operatorname{Tr}_{X}(|\psi\rangle) \equiv \operatorname{Tr}_{X}(|\psi\rangle\langle\psi|)$.

Now we may consider the case that the input $A_{1}$ is maximally entangled with another ancilla $A_{3}$, and similarly for $B_{1}$. Then the input state is

$$
2^{-\left(n_{a}+n_{b}\right) / 2} \sum_{x y}|x\rangle_{A_{1}}|x\rangle_{A_{3}}|y\rangle_{B_{1}}|y\rangle_{B_{3}}|\psi\rangle_{A_{2} B_{2}}
$$

and the output state is

$$
2^{-\left(n_{a}+n_{b}\right) / 2} \sum_{x y}|y\rangle_{A_{1}}|x\rangle_{A_{3}}|x\rangle_{B_{1}}|y\rangle_{B_{3}}\left|c_{x y}\right\rangle_{A_{2} B_{2}}
$$

The increase in the entanglement for $t$ applications of $U$ is then

$$
\begin{aligned}
\Delta E= & S\left[2^{-\left(n_{a}+n_{b}\right)} \sum_{x y}|y\rangle_{A_{1}}\langle y \mid x\rangle_{A_{3}}\langle x| \operatorname{Tr}_{B_{2}}\left(\left|c_{x y}\right\rangle_{A_{2} B_{2}}\right)\right] \\
& -S\left[\operatorname{Tr}_{B_{2}}\left(|\psi\rangle_{A_{2} B_{2}}\right)\right] \\
= & \left(n_{a}+n_{b}\right)+2^{-\left(n_{a}+n_{b}\right)} \sum_{x y} S\left[\operatorname{Tr}_{B_{2}}\left(\left|c_{x y}\right\rangle_{A_{2} B_{2}}\right)\right] \\
& -S\left[\operatorname{Tr}_{B_{2}}\left(|\psi\rangle_{A_{2} B_{2}}\right)\right] \\
= & \left(n_{a}+n_{b}\right)+2^{-\left(n_{a}+n_{b}\right)} \sum_{x y} \Delta E_{x y} .
\end{aligned}
$$

For sufficiently large $t$, we may obtain $\left(n_{a}+n_{b}\right) / t \approx \bar{C}_{+}^{E}$ with arbitrary accuracy. In addition let $E_{U}^{-}$denote the maximum amount by which $U$ can decrease the entanglement. We therefore obtain the inequality $\Delta E / t \geq$ $\bar{C}_{+}^{E}-E_{U}^{-}$, implying

$$
E_{U}+E_{U}^{-} \geq \bar{C}_{+}^{E}
$$

For the case of two-qubit operations, we may simplify this result. In this case the maximum increase in the entanglement $E_{U}$ is equal to the maximum decrease in the entanglement $E_{U}^{-}$, which may be shown in the following way. Any two-qubit interaction $U$ is equivalent, up to local operations, to an operation of the form [7]

$$
U_{d}=\exp \left[-i\left(\alpha_{1} \sigma_{1} \otimes \sigma_{1}+\alpha_{2} \sigma_{2} \otimes \sigma_{2}+\alpha_{3} \sigma_{3} \otimes \sigma_{3}\right)\right],
$$

where $\sigma_{k}$, for $k \in\{1,2,3\}$ are the Pauli operators. As entanglement capability and classical communication capacity are independent of local operations, we may restrict to operations of this form. It is then simple to show that $U_{d}^{*}=U_{d}^{\dagger}=U_{d}^{-1}$. As discussed in Ref. [7], for any measure of entanglement $E(|\Psi\rangle)=E\left(\left|\Psi^{*}\right\rangle\right)$. This means that, if the operation $U_{d}$ acting on the state $|\Psi\rangle$ generates the maximum increase in entanglement, then this operation performed on the state $U_{d}^{*}\left|\Psi^{*}\right\rangle$ decreases the entanglement by $E_{U}$. Therefore, the operation may decrease the entanglement at least as much as it may increase it. Similarly it is simple to show the converse, and therefore $E_{U}=E_{U}^{-}$. Thus we find that, for two-qubit operations, we have the inequality

$$
2 E_{U} \geq \bar{C}_{+}^{E} .
$$

This means that the sum of the communication capacities in each direction cannot be greater than twice the entanglement capability. As mentioned above, in the case of a CNOT or SWAP operation there is equality. That is, the communication that may be performed in each direction is equal to $E_{U}$, for a total of $2 E_{U}$. It would be convenient if we were able to apply a similar argument to prove the converse. That is, if we were able to decompose any operation creating entanglement into a superposition of classical messages, then we would be able to prove $2 E_{U} \leq \bar{C}_{+}^{E}$ and therefore equality. Unfortunately, it does not appear to be possible to establish such a direct equivalence between classical communication and entanglement generation in this way. Nevertheless we will show that, via a two-qubit operation with an entanglement capability of $E_{U}$, it is possible to increase the communication that can be performed in each direction using an ensemble by $E_{U}$ bits.

We will now describe how to obtain initial ensembles for two-qubit operations such that the Holevo information may be increased by $E_{U}$ under operation $U$. In general, for classical message $i$, Bob receives the reduced density matrix $\rho_{i}$. When message $i$ is encoded with probability $p_{i}$, we have the output ensemble $\mathrm{E}=\left\{p_{i}, \rho_{i}\right\}$. Similarly we will denote the ensemble of pure states shared by Alice and Bob by $\mathcal{E}=\left\{p_{i},\left|\psi_{i}\right\rangle_{A B}\right\}$. We use the subscript $A$ to indicate the entire subsystem held by Alice and $B$ to indicate the entire subsystem held by Bob. The Holevo information $\chi$ for ensemble $\mathbf{E}$ is given by

$$
\chi(\mathrm{E})=S\left(\sum_{i} p_{i} \rho_{i}\right)-\sum_{i} p_{i} S\left(\rho_{i}\right) .
$$

This communication may be achieved via coding over multiple states $[8]$. 
The entanglement-assisted communication capacity in a single direction is [5]

$$
C_{\rightarrow}^{E}=\sup \{R:(R, 0) \text { is achievable by } U\} .
$$

As the two-qubit operation is symmetric between Alice and Bob, all results for this case also apply to communication in the opposite direction, $C_{\leftarrow}^{E}$. BHLS show that $C_{\rightarrow}^{E}$ is given by

$$
C_{\rightarrow}^{E}=\sup _{\mathcal{E}}\left[\chi\left(\operatorname{Tr}_{A} U \mathcal{E}\right)-\chi\left(\operatorname{Tr}_{A} \mathcal{E}\right)\right] .
$$

Similarly to BHLS we adopt the notation convention

$$
\begin{aligned}
U \mathcal{E} & =\left\{p_{i}, U\left|\psi_{i}\right\rangle_{A B}\right\}, \\
\operatorname{Tr}_{A} \mathcal{E} & =\left\{p_{i}, \operatorname{Tr}_{A}\left(\left|\psi_{i}\right\rangle_{A B}\right)\right\} .
\end{aligned}
$$

The reason why the communication rate is of the form of a difference in the Holevo information is that, for any $\epsilon, \delta>0$, for sufficiently large $n$ it is possible to construct $n$ copies of the ensemble $\mathcal{E}$ with fidelity $1-\epsilon$ using communication $n\left[\chi\left(\operatorname{Tr}_{A} \mathcal{E}\right)+\delta\right][9,10]$. In the limit of small $\epsilon$ and $\delta$, this means that $\chi\left(\operatorname{Tr}_{A} \mathcal{E}\right)$ bits are required for construction of each ensemble $\mathcal{E}$. The communication that may be performed after the operation $U$ is then $\chi\left(\operatorname{Tr}_{A} U \mathcal{E}\right)$. As communication $\chi\left(\operatorname{Tr}_{A} \mathcal{E}\right)$ was required to construct the ensemble, the additional communication per operation is as given by Eq. (11).

In order to see how to obtain communication equal to $E_{U}$, note that the second term on the right-hand side of Eq. (9) is the average of the entanglement of the coding states. Therefore, if each of the initial states $\left|\psi_{i}\right\rangle_{A B}$ are chosen such that the entanglement of these states is decreased by the maximum $E_{U}$ by operation $U$, then the second term in (9) will be decreased by $E_{U}$ by the operation. If the first term is constant, then the total communication will be $E_{U}$.

In order to obtain such an ensemble, let us start with an initial state $|\Psi\rangle$ such that the entanglement is decreased by the maximum $E_{U}$ via operation $U$. We then wish to find a set of operations $\left\{V_{i}^{A} V_{i}^{B}\right\}$, where $V_{i}^{A}$ are local operations on Alice's side, $V_{i}^{B}$ are local operations on Bob's side, and $V_{i} \equiv V_{i}^{A} V_{i}^{B}$ commutes with $U$. This means that each state $V_{i}|\Psi\rangle$ will have its entanglement decreased by $E_{U}$ for operation $U$. We also require $\sum_{i} p_{i} \operatorname{Tr}_{A}\left(V_{i}|\phi\rangle\right)$ to be a multiple of the identity for all input states $|\phi\rangle$. Then

$$
\sum_{i} p_{i} \operatorname{Tr}_{A}\left(U V_{i}|\Psi\rangle\right)=\sum_{i} p_{i} \operatorname{Tr}_{A}\left(V_{i} U|\Psi\rangle\right) \propto \mathbb{1} .
$$

This means that the first term on the right side of (9) will be unchanged by the operation $U$, and therefore that the total one-way communication possible is $E_{U}$.

Let us consider the case that, in addition to the two qubits upon which $U$ acts, Alice and Bob each possess one auxiliary qubit. It has been found 11 that the maximal increase in entanglement may be achieved using only one auxiliary qubit on each side, and no improvements are obtained using additional auxiliary qubits. We will label Alice's auxiliary qubit 1, the two qubits upon which $U$ acts that Alice and Bob possess as 2 and 3, respectively, and Bob's auxiliary qubit as 4 . For simplicity, and without loss of generality, we will restrict to operations $U$ given in the form (7). In this case the operators $\sigma_{i}^{(2)} \otimes \sigma_{i}^{(3)}$, for $i \in\{0,1,2,3\}$, commute with $U$. Here we take the notation convention that $\sigma_{0}$ is the identity and the superscripts indicate the qubits upon which these operators act. Similarly the operators $\sigma_{i}^{(1)} \otimes \sigma_{i}^{(4)}$ commute with $U$. Let us then consider the set of 16 operators $\left\{\sigma_{i^{\prime}}^{(1)} \sigma_{i}^{(2)} \sigma_{i}^{(3)} \sigma_{i^{\prime}}^{(4)}\right\}$. Given a single state $|\Psi\rangle$ for which $U$ decreases the entanglement by $E_{U}$, we obtain a set of 16 states $\left\{\sigma_{i^{\prime}}^{(1)} \sigma_{i}^{(2)} \sigma_{i}^{(3)} \sigma_{i^{\prime}}^{(4)}|\Psi\rangle\right\}$ for which the entanglement will be decreased by $E_{U}$. In addition, it is easily shown that, for any two-qubit state $\rho$,

$$
\sum_{i i^{\prime}} \sigma_{i}^{(3)} \sigma_{i^{\prime}}^{(4)} \rho \sigma_{i}^{(3)} \sigma_{i^{\prime}}^{(4)} \propto \mathbb{1} .
$$

This implies that

$$
\sum_{i i^{\prime}} \operatorname{Tr}_{12}\left(\sigma_{i^{\prime}}^{(1)} \sigma_{i}^{(2)} \sigma_{i}^{(3)} \sigma_{i^{\prime}}^{(4)}|\Psi\rangle\right) \propto \mathbb{1} .
$$

Thus we find that, by coding with equal probabilities $p_{i i^{\prime}}=1 / 16$ each of the 16 states $\sigma_{i^{\prime}}^{(1)} \sigma_{i}^{(2)} \sigma_{i}^{(3)} \sigma_{i^{\prime}}^{(4)}|\Psi\rangle$, it is possible to communicate $E_{U}$ bits in one direction using operation $U$.

Next we consider the more complicated case of bidirectional communication. Let $i$ be the classical message encoded by Alice, and $j$ be the classical message encoded by Bob. The output ensemble is then $\mathcal{E}=\left\{p_{i}, q_{j},\left|\psi_{i j}\right\rangle_{A B}\right\}$. We will denote the bidirectional communication that it is possible to perform using ensemble $\mathcal{E}$ by $\chi^{\leftrightarrow}(\mathcal{E})$. We also define

$$
\Delta \chi_{U}^{\leftrightarrow}=\sup _{\mathcal{E}}\left[\chi^{\leftrightarrow}(U \mathcal{E})-\chi^{\leftrightarrow}(\mathcal{E})\right]
$$

We again consider a state $|\Psi\rangle$ such that $U$ decreases the entanglement by the maximum $E_{U}$. Alice encodes via the set of 16 operators $\left\{\sigma_{i^{\prime}}^{(1)} \sigma_{i}^{(2)} \sigma_{i}^{(3)} \sigma_{i^{\prime}}^{(4)}\right\}$, and Bob encodes via the 16 operators $\left\{\sigma_{j^{\prime}}^{(1)} \sigma_{j}^{(2)} \sigma_{j}^{(3)} \sigma_{j^{\prime}}^{(4)}\right\}$. Note that Alice and Bob's operators commute, so the order in which these operators are applied is irrelevant. We therefore have a total ensemble of 256 states

$$
\mathcal{E}=\left\{p_{i i^{\prime}}, q_{j j^{\prime}}, \sigma_{i^{\prime}}^{(1)} \sigma_{i}^{(2)} \sigma_{i}^{(3)} \sigma_{i^{\prime}}^{(4)} \sigma_{j^{\prime}}^{(1)} \sigma_{j}^{(2)} \sigma_{j}^{(3)} \sigma_{j^{\prime}}^{(4)}|\Psi\rangle\right\},
$$

where we take $p_{i i^{\prime}}=q_{j j^{\prime}}=1 / 16$. As each of the operators applied by Alice and Bob commutes with $U$ [for $U$ in the form (7)], the entanglement will be decreased by $E_{U}$ for all of these states.

For this type of bidirectional ensemble, the bidirectional communication that it is possible to perform is given by [6]

$$
\chi^{\leftrightarrow}(\mathcal{E})=\chi^{\rightarrow}\left(\operatorname{Tr}_{A} \mathcal{E}\right)+\chi^{\leftarrow}\left(\operatorname{Tr}_{B} \mathcal{E}\right),
$$


where we have defined

$$
\begin{aligned}
& \chi^{\rightarrow} \mathrm{E}=\sum_{j} q_{j}\left[S\left(\sum_{i} p_{i} \rho_{i j}\right)-\sum_{i} p_{i} S\left(\rho_{i j}\right)\right], \\
& \chi^{\leftarrow} \mathrm{E}=\sum_{i} p_{i}\left[S\left(\sum_{j} q_{j} \rho_{i j}\right)-\sum_{j} q_{j} S\left(\rho_{i j}\right)\right],
\end{aligned}
$$

for the ensemble $\mathrm{E}=\left\{p_{i}, q_{j}, \rho_{i j}\right\}$.

Now using the relation (15), we find that

$$
\sum_{i i^{\prime}} \operatorname{Tr}_{12}\left(\sigma_{i^{\prime}}^{(1)} \sigma_{i}^{(2)} \sigma_{i}^{(3)} \sigma_{i^{\prime}}^{(4)} \sigma_{j^{\prime}}^{(1)} \sigma_{j}^{(2)} \sigma_{j}^{(3)} \sigma_{j^{\prime}}^{(4)}|\Psi\rangle\right) \propto \mathbb{1}
$$

and

$$
\sum_{j j^{\prime}} \operatorname{Tr}_{34}\left(\sigma_{i^{\prime}}^{(1)} \sigma_{i}^{(2)} \sigma_{i}^{(3)} \sigma_{i^{\prime}}^{(4)} \sigma_{j^{\prime}}^{(1)} \sigma_{j}^{(2)} \sigma_{j}^{(3)} \sigma_{j^{\prime}}^{(4)}|\Psi\rangle\right) \propto \mathbb{1} .
$$

These relations mean that the first terms in the sums in (20) and (21) are constant under the action of operation $U$. As the entanglement $S\left(\rho_{i j}\right)$ decreases by $E_{U}$ for each state, we find that the total communication that it is possible to perform in each direction using the ensemble is increased by $E_{U}$, for a total increase of $2 E_{U}$.

Thus we have shown the sequence of inequalities $\bar{C}_{+}^{E} \leq$ $2 E_{U} \leq \Delta \chi_{\overleftrightarrow{U}}$. From Ref. [6], the same results are obtained for asymptotically perfect communication as for

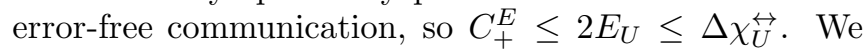
conjecture that, similarly to the case for communication in a single direction [9, 10], we may obtain $n$ copies of the ensemble of states $\mathcal{E}$ using bidirectional communication of $n\left[\chi^{\leftrightarrow}(\mathcal{E})+\delta\right]$. In the limit of large $n$, this would imply that the ensemble $\mathcal{E}$ may be constructed using bidirectional communication per ensemble $\chi^{\leftrightarrow}(\mathcal{E})$. Given this conjecture, we may achieve an average bidi-

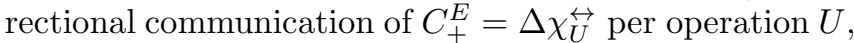
using a scheme equivalent to that considered by BHLS for the case of communication in a single direction. Together with the above results, this would imply that $C_{+}^{E}$ is equal to twice the entanglement capability of the operation. That is, we may communicate $E_{U}$ bits in each direction per operation.

In summary, we have shown that any two-qubit operation $U$ can increase the communication that may be performed using an ensemble by an amount equal to the entanglement capability of $U$. This applies both to cases of communication in just one direction and communication in both directions simultaneously. Furthermore, if the conjecture that the initial pure-state ensemble $\mathcal{E}$ may be constructed using as much classical communication as can be performed using this ensemble (in the limit of a large number of samples) is valid, then the total communication that may be performed in each direction per operation equals the entanglement capability $E_{U}$ in the limit of a large number of operations. Even if this conjecture is not valid (which would be very interesting in itself), we have established that, for arbitrary $U$, there exists $\mathcal{E}$ such that the communication that can be performed using this ensemble increases by $E_{U}$ after application of $U$ to $\mathcal{E}$.

Acknowledgments: This project has been supported by an Australian Research Council Large Grant.
[1] C. H. Bennett et al., Phys. Rev. A 54, 3824 (1996).

[2] D. Collins, N. Linden, and S. Popescu, Phys. Rev. A 64, 032302 (2001).

[3] J. Eisert et al., Phys. Rev. A 62, 052317 (2000).

[4] C. H. Bennett and S. J. Wiesner, Phys. Rev. Lett. 69, 2881 (1992).

[5] C. H. Bennett et al., quant-ph/0205057 (2002).

[6] D. W. Berry and B. C. Sanders, quant-ph/0207065 (2002).
[7] B. Kraus and J. I. Cirac, Phys. Rev. A 63, 062309 (2001).

[8] A. S. Holevo, IEEE Trans. Inf. Theory 44, 269 (1998).

[9] Ref. [5] cites a personal communication by P. W. Shor and a paper in preparation by A. W. Harrow.

[10] D. W. Berry and B. C. Sanders, quant-ph/0209093 (2002).

[11] M. S. Leifer, L. Henderson, and N. Linden, quant$\mathrm{ph} / 0205055$ (2002). 